\title{
An Improved Compiler Directed Power Optimization for Disk Based Systems using Back Propagation Neural Networks
}

\author{
G. Ravikumar and N. Nagarajan
}

\begin{abstract}
The performance of the parallel disk systems is highly affected by excessive power consumption. Hence, various researches are being done in the areas of energy optimization of such systems. Because of the extensive growth and development of computer applications, there has been a huge transformation in the disk subsystem, which mainly includes larger number of disks with higher storage capacities and rotational speeds. Hence, Disk power management has become an essential area of research in recent years as it utilizes very high power. An advanced compiler-directed disk power management approach is presented in this paper which exploits disk access approaches for minimizing energy consumption. This paper examines the various disk access techniques and chooses the optimal approach which would offer better overall performance of the disks via Back Propagation neural network technique which is trained using Modified Levenberg Morquat learning. The experimental evaluation shows that the proposed technique offers better power consumption than the traditional approaches.
\end{abstract}

Keywords--- Modified Levenberg Morquat learning, Hard Disk Drives, Compiler-directed Disk Power Management, Back Propagation Neural Network

\section{INTRODUCTION}

$\mathrm{D}$ ISK power management [1, 2] has been widely investigated in the background of single disk systems, especially for the laptop/ desktop environment.

Minimizing power consumption is becoming a vital issue for high-end cluster/server based systems. These systems are radically different from embedded systems where most of the prior power-related studies appeared. In embedded systems, power research is usuallymotivated by the need of enhancing battery lifetime, in high-end computing both economic and environmental factors play avital role. Especially, recent research [3, 4] shows a huge segment of the system maintenance budget is spent in cooling because of the excessive power consumption of such systems. Moreover, high power consumption, which requires sophisticated power generation and transmission technologies, is known to be harmful to the environment. Therefore, recent years have

G. Ravikumar, Coimbatore Institute of Engineering and Technology, Coimbatore. E-mail: raviciet.research@gmail.com

N. Nagarajan, Coimbatore Institute of Engineering and Technology, Coimbatore, E-mail: swekalnag@ rediffmail.com observed several efforts on minimizing energy consumption of power systems [5, 6].

Majority of the present disks provide diverse power modes of operation such as active and idle. Active mode occurs when the disk is servicing a request and idle mode [7], when it is spinning and not serving a request and one or more low power modes that use less energy than idle. Various researchers have taken up the chance of spinning down the disk during periods of idleness or serving the requests at lower rotational speeds when performance is not a problem. Disk idle periods are considered as a vital factor in those power consumption techniques $[8,9]$.

Most of the traditional techniques are reactive in manner and thus cannot provide efficient results in all the scenarios. The conventional disk power management techniques fail to estimate the disk idleness accurately [10, 11].

Disk access techniques greatly influence the power consumption of the disks [12]. The selection of the appropriate disk access scheduling algorithm has not been effectively taken into consideration for the disk power management.

Artificial Neural Networks (ANNs) computer models inspired by the structure and behavior of real neurons [13] which are used in this paper for choosing the appropriate disk access technique based on the situation.

This paper proposes a compiler-directed disk power management technique which can accurately selects the appropriate disk access technique which in turn provides better power consumption. This technique focuses on developing an accurate and computationally efficient power consumption models for disk-based systems using the compiler directed energy optimization technique.

This proposed approach saves disk power by using the Artificial Neural Network (ANN) to select the most appropriate disk scheduling algorithm and provides effective disk power management.

\section{LITERATURE SURVEY}

Minimizing energy consumption is a vital issue for data centers. Storage is one of the main consumers of energy among various components of a data center. Earlier researches have revealed that the average idle period for a server disk in a data center is very little compared to the time taken to spin down and spin up. This greatly limits the effectiveness of disk power management schemes. Qingbo Zhu et al., [14] in this 
paper proposes several power-aware storage cache management techniques that offer more chances for the fundamental disk power management approaches to save energy. More particularly, an off-line power-aware greedy algorithm is proposed that is better energy-efficient than Belady's off-line algorithm (which minimizes cache misses only). An online power-aware cache replacement algorithm is also proposed in this paper. The trace-driven simulations reveal that, the proposed algorithm saves 16\% more disk energy when compared with Least Recently Used (LRU) algorithm and offers $50 \%$ better average response time for OnLine Transaction Processing (OLTP) I/O workloads. The effects of four storage cache write policies on disk energy consumption are also examined.

Portable media players are vastly using Hard Disk Drives (HDD) to meet their storage needs, but HDDs consume a considerable amount of energy. Hence video frames are prefetched into Dynamic Random Access Memory (DRAM) to facilitate the disk to go into low-power mode; but majority of the mobile systems have limited DRAM, so only very few energy is actually saved in this way. Jaewoo Kim et al., [15] propose two new energy saving approaches: one enhances the use of DRAM in storing prefetched frames, and the other widens this technique by using auxiliary flash memory. The experimental results reveal that deploying a realistic amount of auxiliary flash minimizes disk energy consumption by up to $86 \%$ when compared with traditional prefetching techniques.

In recent times, the requirement for micro hard disk drive that offers high-capacity detachable storage for handheld electronic devices is mounting quickly. The most important issue in the design of seek servo controller in micro disk drives is to diminish power utilization. The input power sent to the seek servo system is used by the transistors of power amplifier and motor coil resistance. Chang-Ik Kang et al., [16] proposed a novel seek servo controller for diminishing the power utilization. In this technique, Fourier decomposition and constrained nonlinear programming are used to find out the optimum seek profile that diminishes the power utilization.

A hybrid hard disk drive that makes use of a non-volatile memory as a cache is increasing attractiveness because of its enhanced consistency and performance. Wanhyung Ryuet al., [17] proposed the design and implementation of a data prefetching method that makes use of flash memory to lessen disk energy utilization in media players. According to the estimated time used to prefetch data into flash memory, selectively decide when to spin up or down the disk in an appropriate way with the intention of reducing disk energy utilization while offering real-time video playback. This method is implemented in MPlayer operating under the platform of the Linux 2.6. Experimental observation shows that the disk energy utilization can be reduced between $35 \%$ and $63 \%$, when a sensible amount of flash memory to a small DRAM can be added.

\section{DISK ACCESS PATTERN EXTRACTION}

The proposed approach uses compiler driven approach for power optimization. The basic necessity for utilizing a compiler in reducing disk power consumption is to identify the way in which the parallel disks are accessed at a high level. The compiler needs the data access pattern of the application code being optimized and disk layout information for the array data as shown in figure 1(a).

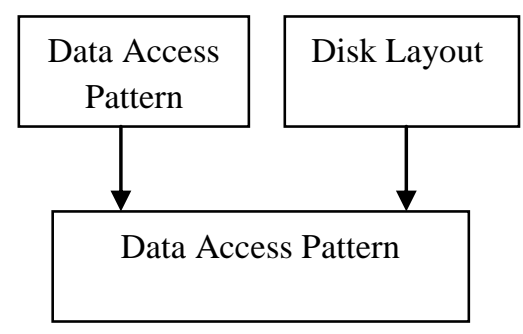

(a)

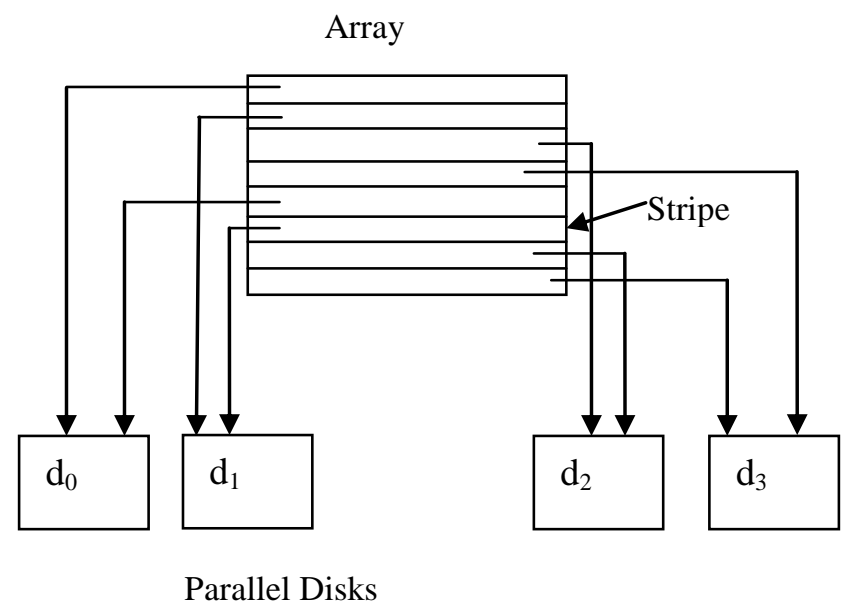

(b)

Figure 1: (a) Determining Disk Access Pattern (b) Striping an Array over Four Disks

The first of these can be attained by examining the application source code. For the second parameter required, the disk layout information is exposed to the compiler. Thus, the compiler will be aware of how array data are striped across the parallel disks and thus can optimizethe source code.

File striping is a method that partitions a large data into small segments and stores these segments on separate disks in a round-robin fashion. This facilitates multiple processes to access various segments of the data concurrently without much disk disputation. In this work, a disk layout of an array is represented using a triplet of the form

$$
\text { (starting_disk, stripe_factor, stripe_size) }
$$

It is observed that various existing file systems and I/O libraries for high-performance computing offer APIs to deliver the disk layout information when the file is created. For instance, in the Parallel Virtual File System (PVFS) [18], the default striping parameters can be altered by setting base (the first I/O node to be used), pcount (stripe factor), and ssize (stripe size) fields of the pvfs_filestat structure. 
Then, the striping information given by the user through this pvfs_filestat structure is passed to the pvfs_open() call's parameter. When building a file from within the application, this layout data can be made accessible to the compiler and moreover the compiler uses this information in conjunction with the data access pattern it extracts to determine the disk access pattern [19].

It is to be noted that, each data array operated by the application is stored in a separate file in the I/O system. As each file can have a distinct triplet, each array can have a distinct disk layout than the others. Thus, the disk access techniques become very vital in optimizing the disk access. Therefore, a novel technique that chooses the best disk access technique through neural networks is proposed in this approach.

\section{A. Proposed Approach}

The patterns in which the disk is accessed play a vital role in the energy optimization. Scheduling algorithms help in identifying the way in which the disk is accessed. This proposed approach focuses on selecting the most suitable scheduling algorithm in relation to the system's load, because operating systems usually use the same scheduling algorithm irrespective of this. To carry out this selection, this approach proposes the use of artificial neural networks.

\section{Disk Seek Algorithms}

The most commonly used strategies for disk scheduling for operating systems [20] are the following:

\section{A. FCFS Planning (First Come, First Served)}

In this type of algorithm the first to arrive is the first to be served, so a request cannot be displaced by the arrival of a new one. There is no reordering of the queue of requests, the positional relationship between the waiting requests is ignored and although it offers a small variance, it is detrimental to requests situated at the end of the queue.

\section{B. SSF Planning (Shortest Seek First)}

In order to lessen the movements of the access method, the disk arm moves to the request nearest to its actual position. It does not consider the order in which requests turn up in the queue, facilitating the central tracks of the disk. This causes the average response time for moderate loads of this approach lower than that of the FCF approach.

Even though this approach carry out well in most cases (it reduces arm movement), it can construct the inanition of certain requests as they are delayed by the advent of new ones that need less arm movement. In order to eradicate the issue of inanition and to make the approach more deterministic a modification of the SSF algorithm is proposed in which requests are dealt in closed batches. Therefore, a set of requests served in its entirety before another is concentrated, and the predictability of the approach is increased considering the average response time of the diverse requests.

\section{SCAN Planning}

The Scan or Elavator approach has been the fundamental of most implemented planning approaches. The main approach of this techniques displacing the disk arm to serve all the requests it identifies in its path, only changing direction when there are no requests in the prevailing direction. Thus, the outer tracks are more visited, so reducing one of the main issues of the (original) SSF algorithm.

This technique has given rise to different modifications among which the $\mathrm{n}$-steps Scan stands out. In this scenario, only the requests which are identified when starting a run are served. The requests which arrive during a run are grouped together and ordered, to be served on the return run.

A second modification has been the C-Scan (Circular Search) algorithm. This approach moves the disk arm from the outer cylinder to the interior. On finalizing the run, it leaps to the request nearest the outer cylinder and initiates again. The third modification has been the Eschenbachapproach. This algorithm moves the disk arm like in the C-Scan but the requests are served according to the arrival time of the sector to the head, i.e. it takes into account rotational delay or latency. In the case of two requests transferring sector positions inside a cylinder, it only serves one in the prevailing arm movement.

\section{Selection of Disk Seek Algorithms using Neural Networks}

ANNs are very appropriate for optimization problems which imply the search for the absolute minimum energy function [25], as shown in figure 2. Object recognition [25], character recognition [25], pattern recognition [25], robot movements [24], modelling cognitive behaviour and predicting financial tends [3], etc., are some of the problems which are very effectively handled by the ANN. In this paper, energy optimization is obtained by the usage of ANN.

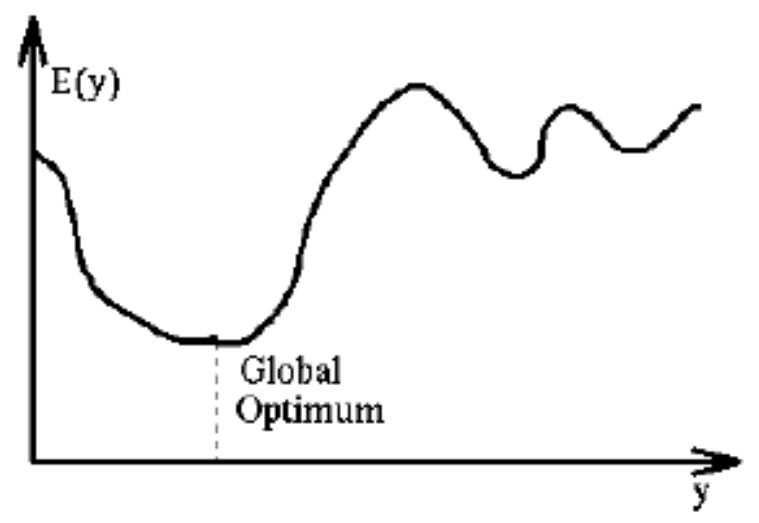

Figure 2: Energy Function

Each approach generates a various number of jumps of the access mechanism, their appropriateness is based on the characteristics of the input / output requests (load) submitted to the system. The jumps show the number of cylinders through which the disk arm passes without stopping to serve an input / output request. These access approach jumps creates delays in the transfer of information, taking up most of the time of an input / output operation. As a result, the selection of the most suitable seek algorithm for each array of input / output requests is essential to globally enhance the performance of the system 
Thus, the performance of the system can be enhanced by using the Back Propagation Neural Network (BPN) to select the most suitable seek algorithm for the load of the system [21].

Figure 3 shows the layout of the back propagation neural network (BPN) in which the processing units (neurons) are interrelated in layers so each neuron transmits its exit signal to every other neuron of the following layer.

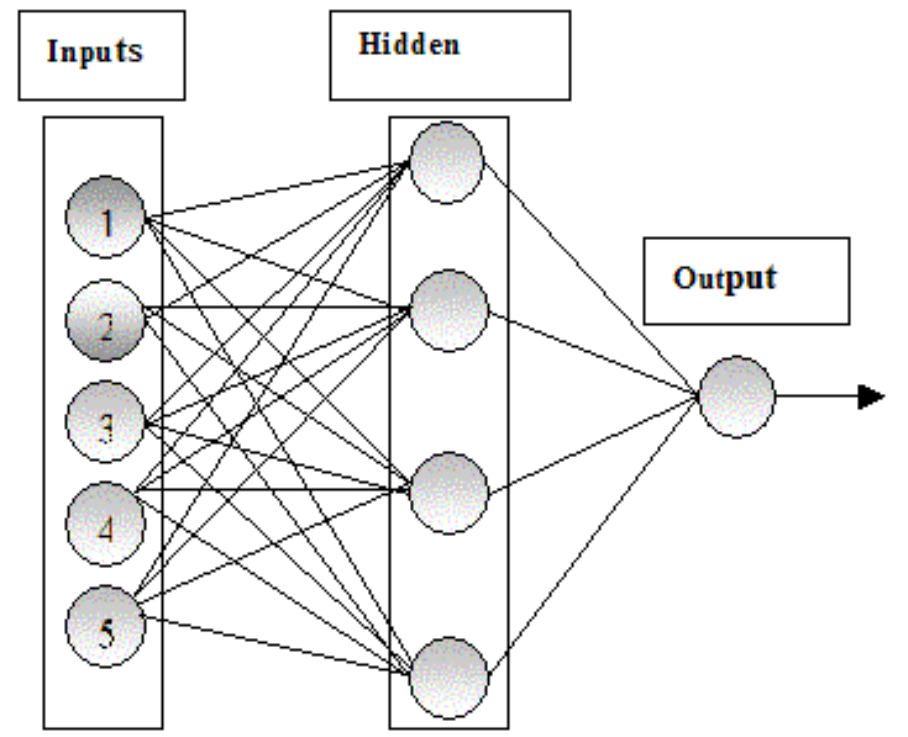

Figure 3: Architecture of Back Propagation Neural Network

BPN is trained using the Modified Levenberg Morquat algorithm. In the BPN algorithm, the performance index F (w) to be minimized is defined as the sum of squared errors between the target outputs and the network's simulated outputs [22].

$$
F(w)=e^{T} e
$$

Where $\mathrm{w}=[\mathrm{w} 1, \mathrm{w} 2, \ldots \mathrm{wN}]$ consists of all weights of the network, $\mathrm{e}$ is the error vector comprising the error for all the training examples.

When training with the LM method, the increment of weights $\Delta \mathrm{w}$ can be obtained as follows:

$$
\Delta w=\left[J^{T} J+\mu I\right]^{-1} J^{T} e
$$

Where $\mathbf{J}$ is the Jacobian matrix, $\mu$ is the learning rate which is to be updated using the $\beta$ depending on the outcome. In particular, $\mu$ is multiplied by decay rate $\beta(0<\beta<1)$ whenever $\mathrm{F}(\mathrm{w})$ decreases, whereas $\mu$ is divided by $\beta$ whenever $\mathrm{F}(\mathrm{w})$ increases in a new step.

The standard LM training process can be illustrated in the following pseudo-codes,

1. Initialize the weights and parameter $\mu(\mu=.01$ is appropriate).

2. Compute the sum of the squared errors over all inputs $F(w)$ 3. Solve (2) to obtain the increment of weights $\Delta \mathrm{w}$
4. Recomputed the sum of squared errors F (w)

Using $\mathrm{w}+\Delta \mathrm{w}$ as the trial $\mathrm{w}$, and judge

IF trial $\mathrm{F}(\mathrm{w})<\mathrm{F}(\mathrm{w})$ in step 2 THEN

$$
\begin{gathered}
w=w+\Delta w \\
\mu=\mu \cdot \beta(\beta=.1)
\end{gathered}
$$

Go back to step 2

ELSE

$$
\mu=\frac{\mu}{\beta}
$$

Go back to step 4

END IF

\section{MODIFICATION OF THE LM METHOD}

Considering performance index is $F(w)=e^{T} e$ using the Newton method we have as

$$
\begin{gathered}
W_{k+1}=W_{k}-A_{k}^{-1} \cdot g_{k} \\
A_{k}=\left.\nabla^{2} \mathrm{~F}(\mathrm{w})\right|_{\mathrm{w}=\mathrm{w}_{\mathrm{k}}} \\
g_{k}=\left.\nabla \mathrm{F}(\mathrm{w})\right|_{\mathrm{w}=\mathrm{w}_{\mathrm{k}}} \\
{[\nabla \mathrm{F}(\mathrm{w})]_{j}=\frac{\partial \mathrm{F}(\mathrm{w})}{\partial w_{j}}} \\
=2 \sum_{i=1}^{N} e_{i}(w) \cdot \frac{\partial e_{i}(w)}{\partial w_{j}}
\end{gathered}
$$

The gradient can write as:

$$
\nabla \mathrm{F}(\mathrm{x})=2 \mathrm{~J}^{\mathrm{T}} \mathrm{e}(\mathrm{w})
$$

Where

$$
\begin{array}{cccc}
\frac{\partial e_{11}}{\partial w_{1}} & \frac{\partial e_{11}}{\partial w_{2}} & \ldots & \frac{\partial e_{11}}{\partial w_{N}} \\
\frac{\partial e_{21}}{\partial w_{1}} & \frac{\partial e_{21}}{\partial w_{2}} & \ldots & \frac{\partial e_{21}}{\partial w_{N}} \\
\vdots & \frac{\partial e_{21}}{\partial w_{2}} & & \frac{\partial e_{21}}{\partial w_{N}} \\
\frac{\partial e_{K P}}{\partial w_{1}} & \frac{1}{\partial w_{2}} & &
\end{array}
$$




$$
J(w)=\left(\begin{array}{cccc}
\frac{\partial e_{11}}{\partial w_{1}} & \frac{\partial e_{11}}{\partial w_{2}} & \cdots & \frac{\partial e_{11}}{\partial w_{N}} \\
\frac{\partial e_{21}}{\partial w_{1}} & \frac{\partial e_{21}}{\partial w_{2}} & \cdots & \frac{\partial e_{21}}{\partial w_{N}} \\
\vdots & \frac{\partial e_{21}}{\partial w_{2}} & \cdots & \frac{\partial e_{21}}{\partial w_{N}}
\end{array}\right)
$$

$\mathrm{J}(\mathrm{w})$ is called the Jacobian matrix. Next we want to find the Hessian matrix. The $\mathrm{k}, \mathrm{j}$ elements of the Hessian matrix yields as:

$$
\begin{gathered}
{\left[\nabla^{2} \mathrm{~F}(\mathrm{w})\right]_{\mathrm{k}, \mathrm{j}}=\frac{\partial \mathrm{F}(\mathrm{w})}{\partial w_{k} \partial w_{j}}} \\
{\left[\nabla^{2} \mathrm{~F}(\mathrm{w})\right]_{\mathrm{k}, \mathrm{j}}=2 \sum_{i=1}^{N}\left\{\frac{\partial e_{i}(w)}{\partial w_{k}} \frac{\partial e_{i}(w)}{\partial w_{j}}+e_{i}(w) \frac{\partial^{2} e_{i}(w)}{\partial w_{k} \partial w_{j}}\right\}}
\end{gathered}
$$

The Hessian matrix can then be expressed as follows:

$$
\nabla^{2} \mathrm{~F}(\mathrm{w})=2 \mathrm{~J}^{\mathrm{T}}(\mathrm{W}) \cdot J(W)+S(W)
$$

Where

$$
S(W)=\sum_{i=1}^{N} e_{i}(w) \cdot \nabla^{2} e_{i}(w)
$$

If $\mathrm{S}(\mathrm{w})$ is small, hessian matrix is approximated as

$$
\nabla^{2} \mathrm{~F}(\mathrm{w})=2 \mathrm{~J}^{\mathrm{T}}(\mathrm{W}) \cdot J(W)
$$

Using (12) and (4) Gauss-Newton method is obtained as:

$$
\begin{gathered}
\left.W_{k+1}=W_{k}-\left[2 J^{T}\left(w_{k}\right) \cdot J\left(w_{k}\right)\right]^{-1} 2 J^{T}\left(w_{k}\right) e\left(w_{k}\right)\right] \\
\left.\cong W_{k}-\left[J^{T}\left(w_{k}\right) \cdot J\left(w_{k}\right)\right]^{-1} J^{T}\left(w_{k}\right) e\left(w_{k}\right)\right]
\end{gathered}
$$

The advantage of Gauss-Newton is that it does not require calculation of second derivatives.

There is a problem the Gauss-Newton method is the matrix $H=J^{T} J$ may not be invertible. This can be overcome by using the following modification. Hessian matrix can be written as:

$$
G=H+\mu I
$$

Suppose that the eigenvalues and eigenvectors of $\mathrm{H}$ are $\left\{\lambda_{1}, \lambda_{2}, \ldots, \lambda_{n}\right\}$ and $\left\{z_{1}, z_{2}, \ldots, z_{n}\right\}$. Then:

$$
\begin{aligned}
G z_{i} & =[H+\mu I]_{z_{i}} \\
& =H z_{i}+\mu z_{i} \\
& =\lambda_{i} z_{i}+\mu z_{i} \\
& =\left(\lambda_{i}+\mu\right) z_{i}
\end{aligned}
$$

Therefore the eigenvectors of $\mathrm{G}$ are the same as the eigenvectors of $\mathrm{H}$, and the eigen values of $\mathrm{G}$ are $\left(\lambda_{i}+\mu\right)$. The matrix $\mathrm{G}$ is positive definite by increasing $\mu$ until $\left(\lambda_{i}+\mu\right)>0$ for all $\mathrm{i}$ therefore the matrix will be invertible. This leads to Levenberg-Marquardt algorithm

$$
\begin{gathered}
\left.w_{k+1}=w_{k}-\left[J^{T}\left(w_{k}\right) J\left(w_{k}\right)+\mu I\right]^{-1} J^{T}\left(w_{k}\right) e\left(w_{k}\right)\right] \\
\left.\Delta w_{k}=\left[J^{T}\left(w_{k}\right) J\left(w_{k}\right)+\mu I\right]^{-1} J^{T}\left(w_{k}\right) e\left(w_{k}\right)\right]
\end{gathered}
$$

As known, learning parameter, $\mu$ is illustrator of steps of actual output movement to desired output. In the standard LM method, $\mu$ is a constant number. This paper modifies LM method using $\mu$ as:

$$
\mu=0.01 e^{T} e
$$

Where e is a $\mathrm{k} \times 1$ matrix therefore $e^{T} e$ is a $1 \times 1$, therefore, $\left[J^{T} J+\mu I\right]$ is invertible.

Therefore, if actual output is far than desired output or similarly, errors are large so, it converges to desired output with large steps. Likewise, when measurement of error is small then, actual output approaches to desired output with soft steps. Therefore error oscillation reduces greatly.

Thus, the most suitable disk access technique is obtained by using neural network. And moreover, the disk access patterns are also obtained [19].

\section{PROACTIVE Disk Power Management}

Thus, disk access patterns are obtained from the above mentioned technique. Then, the compiler can insert clear disk power management calls (instructions) in suitable places in the source code. The principle of these instructions differs depending on the original disk capabilities (for instance, Traditional Power Management (TPM) versus Dynamic Rotations Per Minute (DRPM). For TPM disks, spin_up() and spin_down() calls are used. The format of the spin_down() call is given as follows:

$$
\text { Spin_down }\left(d_{i}\right)
$$

Where $d_{i}$ is the disk ID. As a disk access pattern predictsboth idle and active times, this datacan be utilized topreactivate disks that have been spun down by a spin_down() call. In order toobtain the suitable point in the code to start spinning up the disk, spin-up time (delay) of the disk is considered (that is, the time taken for the disk to reach its full speed where it can perform read/ write activity) [19]. Especially, the number of loop iterations before which the spin-up to be inserted is computed as

$$
Q_{s u}=\left[\frac{T_{s u}}{s+T_{m}}\right]
$$

Where $Q_{s u}$ denotes the preactivation distance (in terms of loop iterations), $T_{s u}$ represents the expected spin-up time (in cycles), $T_{m}$ denotes the overhead incurred by a spin_up call, and $\mathrm{s}$ denotes the number of cycles in the shortest path through the loop body. It is to be observed that $T_{s u}$ is typically much larger than $\mathrm{s}$. The format of the call that is used to preactivate (spin up) a disk is given as follows:

$$
\text { Spin_up }\left(d_{i}\right)
$$


where, as before, $d_{i}$ is the disk ID. It is to be observed that, if preactivation is not used, a TPM disk is automatically spun up when an access (request) comes, but, in this case, the associated spin-up delay is fully incurred. The purpose of disk preactivation is to eliminate this performance penalty.

\section{EXPERIMENTAL RESULTS}

Setup

This disk power simulator similar to Disk Sim [23] is determined by externally provided disk I/O request traces, which are constructed by the trace generator. The disk energy consumption comprises of all the energy consumptions in both active and idle periods, considering all the states of disks during the whole execution. Also, the performance numbers include all conflicts in accessing the parallel disk system.

Table1: Benchmarks and Their Characteristics

\begin{tabular}{|c|c|}
\hline Benchmarks & $\begin{array}{c}\text { Data Size } \\
(\mathrm{GB})\end{array}$ \\
\hline 168.wupwise & 88.6 \\
\hline 171.swim & 64.7 \\
\hline 172.mgrid & 75.5 \\
\hline 173.applu & 100.6 \\
\hline
\end{tabular}

The performance of the proposed approach is compared with the approaches such as TPM and DRPM for each benchmark code in this experimental suite.

Results

The performance evaluation of the proposed approach is compared with the Traditional Power management systems and DRPM. The energy saving comparison for various power loads are evaluated and compared.

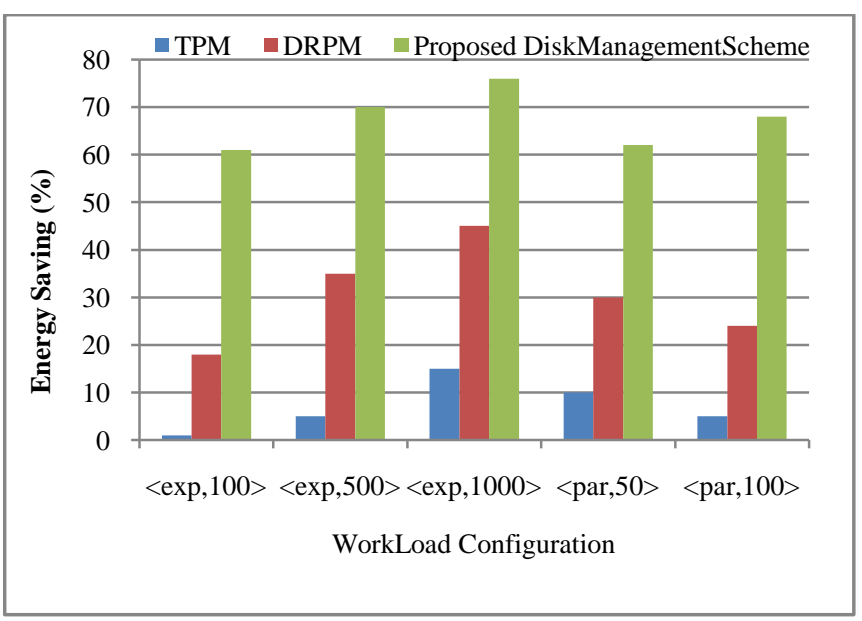

Figure 4: Comparison of Energy Savings for Exponential and Pareto Methods

Figure 4 shows the comparison of the proposed approach with the existing approaches such as TPM and DRPM. It is observed from the graph that for different work load applied to the power management techniques, the energy saving obtained by the proposed approach is very high when compared with TPM and DRPM.

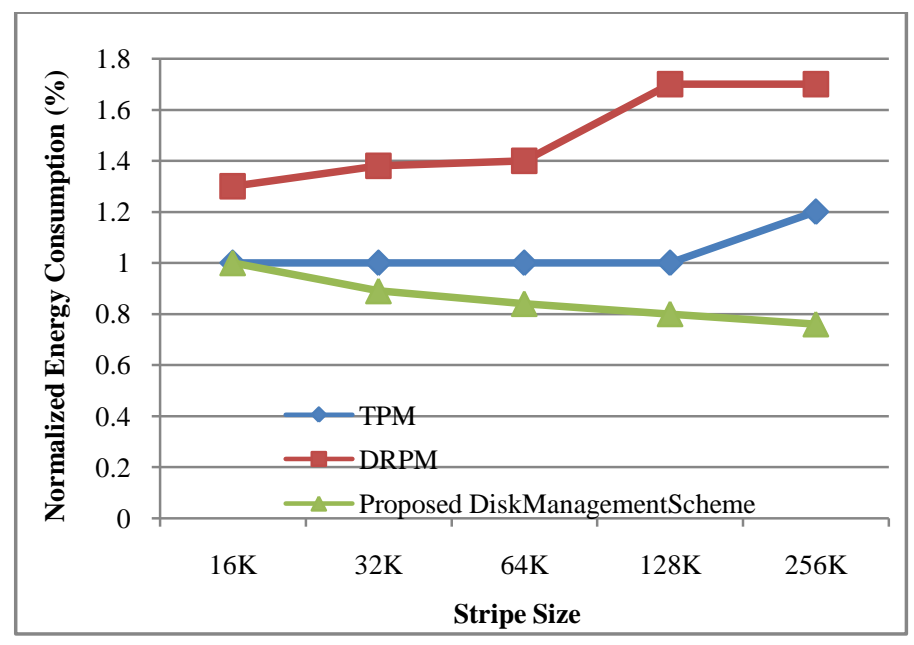

Figure5: Impact of stripe size on energy consumption

Figure 5 shows the normalized energy consumptions with the different stripe sizes. It is clearly observed from the graph that the energy savings brought by the traditional based approaches increase as the stripe size increases. When the stripe size is very small (16 Kbyte), disks do not experience much idleness. Actually, the disk idleness in this scenario becomes so little that even code restructuring cannot take much gain of it. When the stripe size is increased, more disk requests can be serviced by a single stripe, that is, the stripelevel data reuse improves. It is observed that the proposed disk management scheme generates the best savings with 256 Kbyte stripe size.

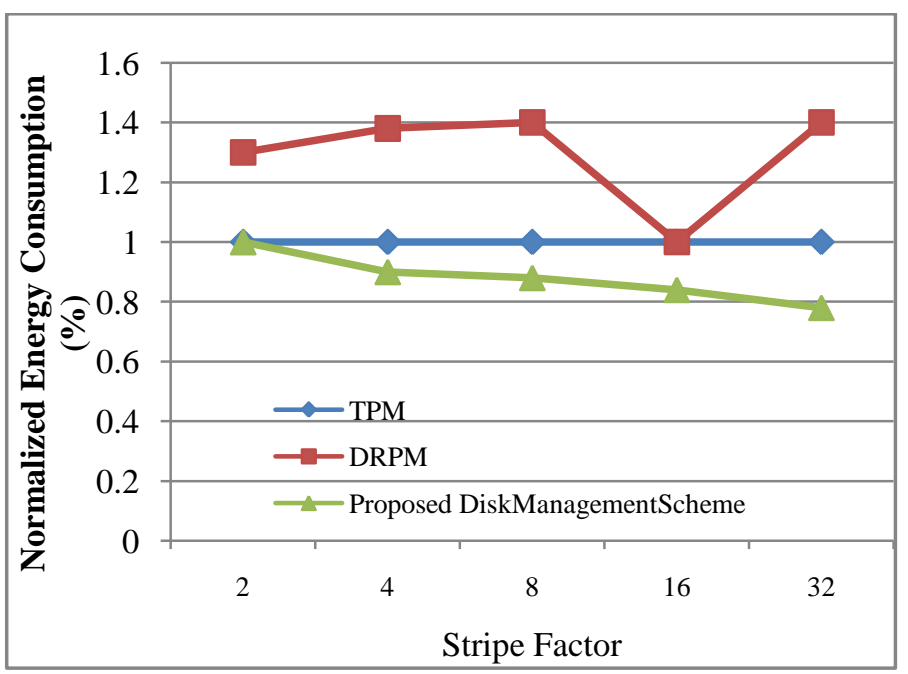

Figure 6: Impact of Stripe Factor on Energy Consumption

Figure 6 shows the normalized energy values under different stripe factors. It is observed that as the number of disks is increased, disk idleness increases, and consequently, the compiler schemes exhibit a better behavior. When the number of disks is very high (32), the disk idleness reaches a very high level. The proposed Disk Management scheme is 
observed to obtain less energy consumption for all the stripe factors.

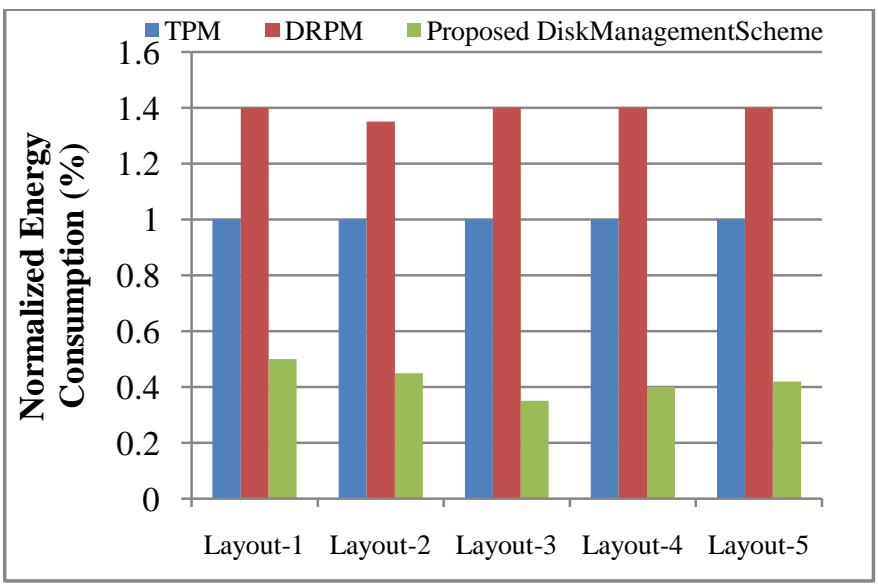

Figure7: Impact of Starting Disk on Energy Consumption

Figure 7 shows the impact of starting disk on the energy consumption. To perform this set of experiments, a random integer number is constructed (for each array in the mgrid benchmark) between 1 and 8 to choose the disk from which the array is striped. Figure 7 shows the results for five such experiments. It is observed from the graph that the proposed scheme consumes less energy when compared with exising TPM and DRPM approaches.

Figure 5, 6 and 7 shows the impact of the stripe size, stripe factor and starting disk on energy consumption. It is observed that the proposed approach has better energy consumption in all the three cases. Thus, the proposed approach out performs the other two approaches in terms of energy consumption.

\section{CONCLUDING REMARKS}

Disk system is a key contributor to total energy consumption of high-end parallel systems. There are several approaches available in the literature to minimize disk power considering idle periods experienced by disks. This paper presents a profile-driven technique to diminish the energy consumption. This technique uses a neural network approach for selecting suitable disk access techniques. BPN neural network approach is used in this approach for selecting the appropriate disk access techniques. The experiment results reveal with this approach greatly reduces energy consumption of original applications significantly. The best energy savings are obtained by a combined scheme that selects a suitable disk access technique. The performance of the proposed approach is enhanced by using Modified Leven berg-Marquardt Method for training the BPN.

\section{REFERENCES}

[1] L. Cai and Y.-H. Lu, "Joint Power Management of Memory and Disk", In Proceedings of the Conference on Design, Automation and Test in Europe, Pp. 86-91, 2005.

[2] L. Cai and Y.H. Lu, "Power Reduction of Multiple Disks Using Dynamic Cache Resizing and Speed Control", In Proceedings of the International Symposium on Low Power Electronics and Design, Pp. 186-190, 2006.
[3] P. Bohrer E. Elnozahy, T. Keller, M. Kistler, C. Lefurgy, C. McDowell, and R. Rajamony, "Power-Aware Computing, chapter The Case for Power Management in Web Servers", Kluwer Academic Publisher, Jan. 2002.

[4] M. Elnozahy, M. Kistler, and R. Rajamony, "Energy Conservation Policies for Web Servers", In Proceedings of the 4th USENIX Symposium on Internet Technologies and Systems, March 2003.

[5] J. Chase, D. Anderson, P. Thackar, A. Vahdat, and R. Boyle, "Managing Energy and Server Resources in Hosting Centers", In Proceedings of the 18th Symposium on Operating Systems Principles, Pp. 103-116, October 2001.

[6] J. Chase and R. Doyle, "Balance of Power: Energy Management for Server Clusters", In Proceedings of the 8th Workshop on Hot Topics in Operating Systems, Pp. 165, May 2001.

[7] S. Gurumurthi, A. Sivasubramaniam, M. Kandemir, and H. Franke, "DRPM: Dynamic Speed Control for Power Management in Server Class Disks," Proc. Int'l Symp. Computer Architecture, Pp. 169-179, June 2003.

[8] F. Douglis, P. Krishnan, and B. Bershad, "Adaptive Disk Spin- Down Policies for Mobile Computers," Proc. Second Symp. Mobile and Location-Independent Computing, Pp. 121-137, 1995.

[9] F. Douglis, P. Krishnan, and B. Marsh, "Thwarting the Power- Hungry Disk,” Proc. Usenix Winter Conf., Pp. 292-306, 1994.

[10] E.N.M. Elnozahy, M. Kistler, and R. Rajamony, "Energy-Efficient Server Clusters", Proc. Second Workshop Power Aware Computing Systems, Feb. 2002.

[11] K. Li, R. Kumpf, P. Horton, and T. Anderson, "A Quantitative Analysis of Disk Drive Power Management in Portable Computers", Proc. Usenix Winter Conf., Pp. 279-292, 1994

[12] David La Red and José Ignacio Peláez, "Optimization of Disk Seek Operations using Neural Networks".

[13] Simon Haykin, Neural Networks: A comprehensive Foundation. Prentice Hall. 1999.

[14] Qingbo Zhu, David, F.M, Devaraj C.F, Zhenmin Li, Yuanyuan Zhou, Pei Cao, "Reducing Energy Consumption of Disk Storage Using PowerAware Cache Management”, Pp. 118, 2004.

[15] Jaewoo Kim, Ahron Yang, Minseok Song, "Exploiting flash memory for reducing disk power consumption in portable media players", IEEE Transactions on Consumer Electronics, Vol. 55 , Pp. 1997 - 2004, 2009.

[16] Chang-Ik Kang, Sang-EunBaek, Jun-Seok Shim, "A new seek servo controller for minimizing power consumption in micro hard disk drives", IEEE Transactions on Magnetics, Vol. 40, Issue: 4, Part: 2, Pp. 3127 - 3129, 2004.

[17] Wanhyung Ryu, Minseok Song, "Design and implementation of a disk energy saving scheme for media players which use hybrid disks", IEEE Transactions on Consumer Electronics, Vol. 56, Issue: 4, Pp. 2382 2386, 2010

[18] R.B. Ross, P.H. Carns, W.B. Ligon III, and R. Latham, "Using the Parallel Virtual File System," http://www.parl.clemson.edupvfs/userguide.html, July 2002.

[19] Seung Woo Son, Guangyu Chen and OzcanOzturk, MahmutKandemir and AlokChoudhary, "Compiler-Directed Energy Optimization for Parallel-Disk-Based Systems", IEEE Transactions On Parallel and Distributed Systems, Vol. 18, No. 9, September 2007.

[20] Deitel H. M, "Introducción a los SistemasOperativos", Addison-Wesley Iberoamericana 1987.

[21] Bing Gong, "A Novel Learning Algorithm of Back-Propagation Neural Network", IITA International Conference on Control, Automation and Systems Engineering 2009.

[22] Amir AbolfazlSuratgar, Mohammad BagherTavakoli, and Abbas Hoseinabadi, "Modified Levenberg-Marquardt Method for Neural Networks Training", World Academy of Science, Engineering and Technology 2005 . 
[23] J.S. Bucy et al., "The DiskSim Simulation Environment Version 3.0 Reference Manual," Technical Report CMU-CS-03-102, CMU, Jan. 2003.

[24] Mammone R, Zeevi Y, "Neural Networks. Theory and Applications", Academic Press Inc., 1991.

[25] Patterson D, "Artificial Neural Networks. Theory and Applications", Prentice Hall Inc., 1995.

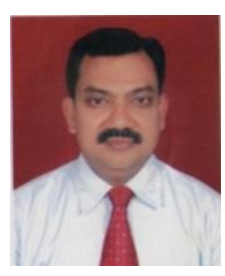

G.Ravikumar received his M.Tech., degree and B.E., degree in Computer Science and Engineering from Bharathidasan and Sastra University respectively. He is currently working as assistant professor in department of Computer Science and Engineering at Coimbatore Institute of Engineering and Technology, Coimbatore. His research interests accumulate in the area of Optimization in parallel disk based system towards energy and power. E-mail: raviciet.research@gmail.com

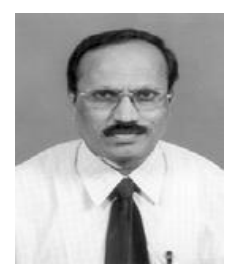

Dr.N.Nagarajan received his B.Tech and M.E. degrees in Electronics Engineering at M.I.T Chennai. He received his $\mathrm{PhD}$ in faculty of information and communication engineering from Anna University, Chennai. He is currently working as Principal in Coimbatore Institute of Engineering and Technology at Coimbatore. He is member of board of study of faculty of information Technology at Anna University, Coimbatore. His specialization includes optical, wireless Adhoc and sensor networks. He is guiding assorted research scholars in power and energy optimization. E-mail: swekalnag@ rediffmail.com. 